\title{
ON THE AUTOMORPHIC FUNCTIONS OF THE GROUP
}

\author{
$\left(0,3 ; l_{1}, l_{2}, l_{3}\right)^{*}$ \\ BY

\section{RICHARD MORRIS}

The chief aim of this paper is to investigate the representation of groups of the type $\left(0,3 ; l_{1}, l_{2}, l_{3}\right) \dagger$ and of the functions belonging to thein, and in particular to show that for no other groups of this type except those already studied $\ddagger$ can the functions which are automorphic for the group be expressed in terms of theta functions which contain explicitly only one parameter the linear transformations of which detine the group in question.

The Riemann surface, of genus $p$, defined by the equation

$$
W^{\nu}=(z-a)^{\alpha}(z-b)^{\beta}(z-c)^{\gamma}(z-d)^{\delta},
$$

in which $\alpha+\beta+\gamma+\delta=k \nu(k=1,2,3), \alpha, \beta, \gamma, \delta$ being each less than $\nu$, depends upon only one irreducible parameter. If the branch-points vary in any way so that at the end of the variation they occupy their original positions (or are permuted in case of equal exponents), the parameter undergoes a certain transformation. Let $u_{i}\left(i=1,2, \ldots, p^{\prime} ; p^{\prime} \leqq p\right)$ denote a set of linearly

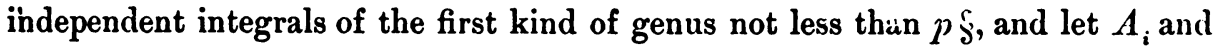
$B_{i}$ denote the results of integrating $u_{i}$ around the paths $a_{i}$ and $b_{i}$ respectively. Then the moduli of periodicity of $u_{i}$ at tine cuts $a_{k}, b_{k}$ are expressible in the form $\| m_{k} A_{i}+n_{k} B_{i}, m_{k}^{\prime} A_{i}+n_{k}^{\prime} B_{i}$. Let $\omega_{i}$ denote the quotient $A_{i}: B_{i}$. Then $\omega_{i}$ may be taken as the parameter of the Riemann surface. Any other such constant, $\omega_{k}$, must then be expressible in terms of $\omega_{i}$, either linearly by means of the bilinear relations among the moduli of the integrals of the first

* Presented to the Society September 7, 1905. Received for publication April 16, 1906.

† See Fricke and Krein, Automorphe Funtionen, vol. 1, p. 353, for this notation.

‡ BURKHARDT, Mathematiscbe Annalen, vol. 42 (1893), p. 185 ; Hutchinson, these Transactions, vol. 3 (1902), p. 1 ; vol. 5 (1904), p. 447 ; Young, these Transuctions, vol. 5 (1901), p. 81.

$\$$ It is evident that $p^{\prime}$ is equal to $\phi(\nu)$ and that $\varphi(v)$ is always even for $\nu>2$, where $\phi(v)$ is the number of values that $q$ inay have, $q$ heing taken as deficed in $\$$. If $p$ is greater than $\phi(\nu)$, then those integrals in excess of $\phi(v)$ in number, which are dependent upon values of $q$ not relatively prime to $\nu$, will be of genus less than $p$ and need not be considered in this connection.

II See Automorphe Functionen, vol. 2, p. 135. 
kind, or transcendentally by the vanishing of certain theta formulæ. For each monodrony of the branch-points of the Riemann surface the parameter $\omega_{i}$ undergoes a linear transformation, and the totality of these form a group $\Gamma$.

In the case of the elliptic Riemann surface, every function of $\omega$, as defined above, which is automorphic for the group can be expressed by means of the elliptic theta functions with zero argument and modulus $\omega$.

The hyperelliptic surfaces $W^{3}=(z-a)(z-b)(z-c)^{2}(z-d)^{2}$ and $W^{4}=(z-a)(z-b)^{2}(z-c)^{2}(z-d)^{3}$ were first considered from this point of view by BurkhardT,* and were further investigated by Hutchinson * and Young * respectively. Hutchinson * has also investigated the surface $W^{6}=(z-a)^{2}(z-b)^{2}(z-c)^{3}(z-d)^{5}$. In these cases, the moduli for the integrals of the first kind of genus $p$ are all linearly expressible in terms of a single parameter $\omega$ which undergoes a linear transformation for each monodromy of the branch-points. Every function of $\omega$ which is automorphic for the group of linear transformations so determined for each of the above cases is expressible in terms of hyperelliptic theta functions.

In the recently published work of FrICKE and KLEIN on automorphic functions $\nmid$ a section is devoted to the general consideration of automorphic functions which are associated in a similar manner with the Riemann surface (1). and the suggestion is there made that such functions would be expressible in terms of the theta functions whose moduli are those of the normal integrals of the first kind for that surface. The authors speak of the desirability of studying along this line other cases besides those investigated by BurkhardT. Following this suggestion the present writer found, on examining a number of cases, that the moduli $\omega_{i}$ are not all expressible linearly in terms of one of them.

The problem then is: What are the cases in which all the moduli of the $p^{\prime}$ integrals of the first kind of genus $p$ are linearly expressible in terms of a single parameter? We shall show that there are none besides those referred to above. The proof consists of two parts.

I. It is shown that there is a finite number of cases in which all the $\omega_{i}^{\prime}$ s reduce linearly to a single one. This is done by showing that any $\omega_{i}$ cannot be connected linearly with more than three others in case two of the exponents $\alpha, \beta, \gamma, \delta$ are equal, or with more than seven others in case these exponents are all different, and hence the $p^{\prime}$ parameters $\omega_{i}$ can reduce linearly to a single one only when $p^{\prime}$ does not exceed 4 or 8 in the respective cases.

II. It is shown that this finite number of cases reduces to those already known, by assuming that the automorphic functions are uniform, thus placing conditions upon the multipliers of the generating substitutions of the group.

*Loc. cit.

† See Automorphe Functionen, vol. 2, p. 131 et seq. 


\section{PART I.}

$$
\S 1 . \alpha^{\prime}+\beta^{\prime}+\gamma^{\prime}+\delta^{\prime}=\nu \text { or } 3 \nu \text {. }
$$

Let $q$ be any positive number relatively prime to $\nu$ and less than $\nu$, and assume the relations given in the equations

$$
q \alpha=A \nu+\alpha^{\prime}, \quad q \beta=B \nu+\beta^{\prime}, \quad q \gamma=C \nu+\gamma^{\prime}, \quad q \delta=D \nu+\delta^{\prime},
$$

in which $A, B, \ldots, \alpha^{\prime}, \beta^{\prime}, \ldots$ are positive integers, and $\alpha^{\prime}, \beta^{\prime}, \ldots$ each less than $\nu$. Then all the integrals of the first kind of genus $p$ can be expressed in the form

$$
u_{i}=\int \frac{(z-a)^{A}(z-b)^{B}(z-c)^{C}(z-d)^{D} d z}{W^{q^{2}}}=\int \frac{F(z) d z}{W^{i}},
$$

unless $\alpha^{\prime}+\beta^{\prime}+\gamma^{\prime}+\delta^{\prime}=\nu$. In this event, however, there is another value of $q$, namely $q_{1} \equiv-q$ (mod. $\nu$ ), such that the sum of the corresponding $\alpha^{\prime}, \beta^{\prime}, \gamma^{\prime}, \delta^{\prime}$ is $3 \nu$, giving two integrals of the first kind of the form

$$
u_{k}=\int \frac{F_{1}(z) d z}{W^{q_{1}}}, \quad \text { and } \quad u_{k+1}=\int \frac{z \cdot F_{1}(z) d z}{W^{q_{1}}} .
$$

For, substituting $q_{1}=\nu-q$ for $q$ in equations (2), we have

$$
\begin{array}{ll}
(\nu-q) \alpha=A^{\prime} \nu+\alpha^{\prime \prime}, & (\nu-q) \beta=B^{\prime} \nu+\beta^{\prime \prime}, \\
(\nu-q) \gamma=C^{\prime} \nu+\gamma^{\prime \prime}, & (\nu-q) \delta=D^{\prime} \nu+\delta^{\prime \prime},
\end{array}
$$

and by adding equations (2) and (5),

$$
\nu x=\left(A+A^{\prime}\right) \nu+\alpha^{\prime}+\alpha^{\prime \prime}, \quad \nu \beta=\left(B+B^{\prime}\right) \nu+\beta^{\prime}+\beta^{\prime \prime}, \quad \text { etc. }
$$

Since $\alpha^{\prime}, \alpha^{\prime \prime}, \ldots$ are positive and less than $\nu$, it follows that

$$
\alpha^{\prime}+\alpha^{\prime \prime}=\nu, \quad \beta^{\prime}+\beta^{\prime \prime}=\nu, \quad \gamma^{\prime}+\gamma^{\prime \prime}=\nu, \quad \delta^{\prime}+\delta^{\prime \prime}=\nu,
$$

\begin{tabular}{|c|c|c|c|c|c|c|}
\hline & $n_{1}$ & $a_{2}$ & $\cdots$ & $b_{1}$ & $b_{2}$ & \\
\hline$u_{k}$ & $m_{1} A_{k}+n_{1} B_{k}$ & $m_{2} A_{k}+n_{2} B_{k}$ & $\cdots$ & $m_{1}^{\prime} A_{k}+n_{1}^{\prime} B_{k}$ & $m_{2}^{\prime} A_{k}+n_{2}^{\prime} B_{k}$ & \\
\hline & $m_{1} A_{k+1}+n_{1} B_{k+1}$ & $m_{2} A_{k+1}+n_{2} B_{k+1}$ & . . & $m_{1}^{\prime} A_{k+1}+n_{1}^{\prime} B_{k+1}$ & $m_{2}^{\prime} A_{k+1}+n_{2}^{\prime} B_{k+1}$ & $\ldots$ \\
\hline
\end{tabular}

whence

$$
\left(\alpha^{\prime}+\beta^{\prime}+\gamma^{\prime}+\delta^{\prime}\right)+\left(\alpha^{\prime \prime}+\beta^{\prime \prime}+\gamma^{\prime \prime}+\delta^{\prime \prime}\right)=4 \nu,
$$

and since $\alpha^{\prime}+\beta^{\prime}+\gamma^{\prime}+\delta^{\prime}=\nu$, therefore $\alpha^{\prime \prime}+\beta^{\prime \prime}+\gamma^{\prime \prime}+\delta^{\prime \prime}=3 \nu$.

It is evident from the following that there is no linear relation between the moduli of the two integrals $u_{k}$ and $u_{k+1}$. Let $A_{k}, A_{k+1}$ and $B_{k}, B_{k+1}$ denote the values of these integrals when integrated along the period paths $a_{1}$ and $b_{1}$ respectively. Since the denominators of these integrals are the same in both, the table of moduli of periodicity for $u_{k}$ and $u_{k+1}$ will have the following form : 
in which the coefficients $m_{i}, m_{i}^{\prime}, n_{i}, n_{i}^{\prime}$ are functions of the $\nu$ th roots of unity, and $n_{1}=-1, m_{1}=0, m_{1}^{\prime}=1, n_{1}^{\prime}=0$. The bilinear relation among these moduli is

$\sum_{i=1}^{n}\left[\left(m_{i} A_{k}+n_{i} B_{k}\right)\left(m_{i}^{\prime} A_{k+1}+n_{i}^{\prime} B_{k+1}\right)-\left(m_{i} A_{k+1}+n_{i} B_{k+1}\right)\left(m_{i}^{\prime} A_{k}+n_{i}^{\prime} B_{k}\right)\right]=0$

which reduces to

$$
\sum_{i=1}^{p}\left(m_{i} n_{i}^{\prime}-m_{i}^{\prime} n_{i}\right)\left(A_{k} B_{k+1}-A_{k+1} B_{k}\right)=0 .
$$

If the second of these factors vanishes, we get $A_{k}: B_{k}=A_{k+1}: B_{k+1}$. But this cannot be, for then two rows in the table of moduli would difter only by a factor of proportionality, which is evidently impossible. Hence

$$
\sum_{i=1}^{p}\left(m_{i} n_{i}^{\prime}-m_{i}^{\prime} n_{i}\right)=0
$$

and the relation becomes identity. Accordingly $\omega_{k}$ is not linearly connected with $\omega_{k+1}$. Hence

THEOREM I. In case the sum of the residues of $q x, q \beta, q \gamma, q \delta$ tuken mod. $\nu$ is $\nu$ ( $q$ being relatively prime to $\nu)$, there always exist two integrals of the first kind $u_{k}, u_{k+1}$ such that the corresponding iararmeters $\omega_{k}, \omega_{k+1}$ are linearly inclependent.

In what follows we shall therefore consider only those cases in which the sum of the exponents $\alpha, \beta, \gamma, \delta$ is $2 \nu$, and also the sum of the exponents $\alpha^{\prime}, \beta^{\prime}, \gamma^{\prime}, \delta^{\prime}$. obtained by multiplying $\alpha, \beta, \gamma, \delta$ by any integer $q$ and reducing mod. $\nu$ is $2 \nu$.

The different cases to be investigated are the following:

$$
\begin{aligned}
& \text { Cluss 1. } x=\beta=\gamma=\delta \text {. } \\
& \text { “2. } \alpha=\beta=\gamma \neq \delta \text {. } \\
& \text { “3. } x=\delta, \beta=\gamma, x \neq \beta, x+\beta=\nu \text {. } \\
& \text { “4. } \beta=\gamma, x \neq \beta \neq \delta \text {. } \\
& \text { “5. } \alpha \neq \beta \neq \gamma \neq \delta \text {. }
\end{aligned}
$$

\section{§ 2. The monodiomy group of the Riemann surface.}

The monodromy group for each of the classes, indicated at the end of the previous section, is next obtained.

$$
\text { Class 1. } \alpha=\beta=\gamma=\delta \text {. }
$$

Since $x+\beta+\gamma+\delta=2 \nu, \alpha=\nu / 2$. The equation (1) thus reduces to that of the elliptic Riemann surface. 


$$
\text { Class 2. } \alpha=\beta=\gamma \neq \delta \text {. }
$$

A diagram for the Riemann surface in this class is given in Fig. 1, in which the branch-points are connected by branch-lines with the point at infinity.

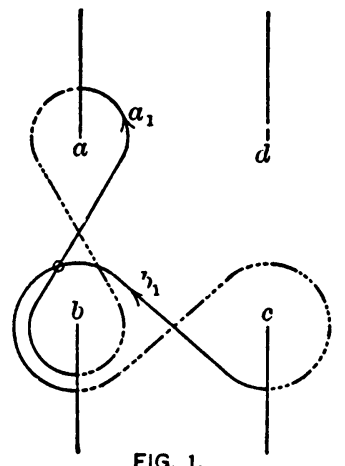

FIG. 1 .

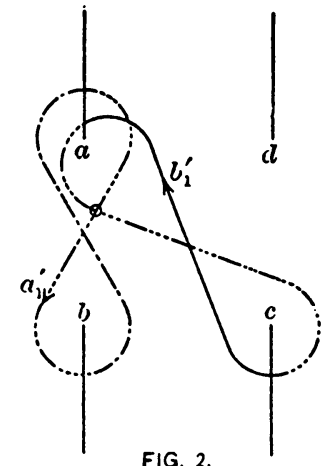

FIG. 2 .

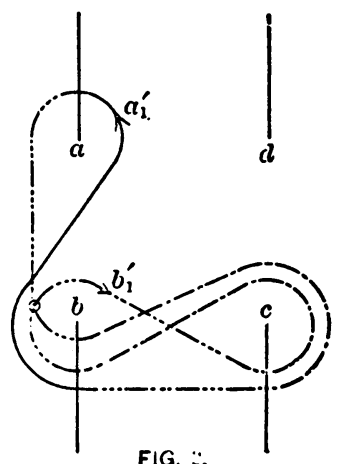

FIG. :

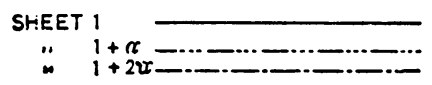

Let

$$
u_{1}=\int \frac{d z}{W}
$$

be an integral of the first kind, and $A_{1}, B_{1}$ the values of $u_{1}$ when integrated along $a_{1}$ and $b_{1}$ respectively, and put $c=e^{2 \pi i / \nu}$. Also, let $\alpha_{i}$ be the value of $u_{1}$ when integrated from the point in the $i$ th sheet corresponding to a given fixed value $z=z_{0}$ along a path circumscribing the branch-point $a$ by a positive rotation and returning in the new sheet to the point corresponding to the initial value $z_{0}$ of $z$. Let $\beta_{i}, \gamma_{i}$ and $\delta_{i}$ be similarly defined.

If

then

$$
\alpha_{1}=\frac{d z}{W},
$$

$$
\alpha_{1+a}=\int \frac{d z}{\sigma^{\alpha} W}=\sigma^{-\alpha} \alpha_{1},
$$

the paths of integration being as just defined, and hence,

$$
A_{1}=\alpha_{1}-\beta_{1} \text { and } B_{1}=\beta_{1}-\gamma_{1} .
$$

We next determine the effect on the moduli $A_{1}, B_{1}$ of a monudromy of the branch-points. In the first place imagine the line joining the branch-points $a$ and $b$ rotated positively about its middle point until $a$ and $b$ are interchanged. The paths $a_{1}$ and $b_{1}$ will be deformed at the same time into $a_{1}^{\prime}$ and $b_{1}^{\prime}$ as indicated in Fig. 2. Denote by $A_{1}^{\prime}$ and $B_{1}^{\prime}$ the result of integrating $u_{1}$ along the new paths. It is readily seen that these integrals take the form

$$
A_{1}^{\prime}=\beta_{1+a}-\alpha_{1+a}=-\sigma^{-\alpha} A_{1}, \quad B_{1}^{\prime}=\alpha_{1}-\gamma_{1}=A_{1}+B_{1} .
$$


If now $\omega_{1}=A_{1} / B_{1}$, we get

whence

$$
\frac{A_{1}^{\prime}}{B_{1}^{\prime}}=-\sigma^{-a} A_{1},
$$

$$
\omega_{1}^{\prime}=\frac{-\sigma^{-a} \omega_{1}}{\omega_{1}+1}
$$

We denote this substitution on $\omega_{1}$ by $V_{1}$.

Similarly, if the line joining $b$ and $c$ is rotated positively about its middle point until $b$ and $c$ are interchanged, the paths $a_{1}$ and $b_{1}$ are deformed at the same time into $a_{1}^{\prime}$ and $b_{1}^{\prime}$ as shown in Fig. 3. The integrals along the new paths take the form

and

$$
A_{1}^{\prime}=\alpha_{1}+\beta_{1+a}-\gamma_{1+a}-\beta_{1}=A_{1}+\sigma^{-a} B_{1},
$$

$$
B_{1}^{\prime}=\gamma_{1+a}-\beta_{1+a}=-\sigma^{-a} B_{1} \text {. }
$$

Dividing $\mathbf{A}_{1}^{\prime}$ by $B_{1}^{\prime}$, we get

$$
\omega_{1}^{\prime}=-\sigma^{\alpha} \omega_{1}-1
$$

which substitution is denoted by $S_{1}$.

Any other substitution produced by a monodromy of the branch-points may be obtained by a suitable combination of the substitutions $S_{1}$ and $V_{1}$.

$$
\text { Class 3. } \alpha=\delta, \beta=\gamma, \alpha \neq \beta \text {. }
$$

A diagram for the Riemann surface in this class is given in Fig. 4, where the branch-points, as in Class 2, are connected with the point at infinity.

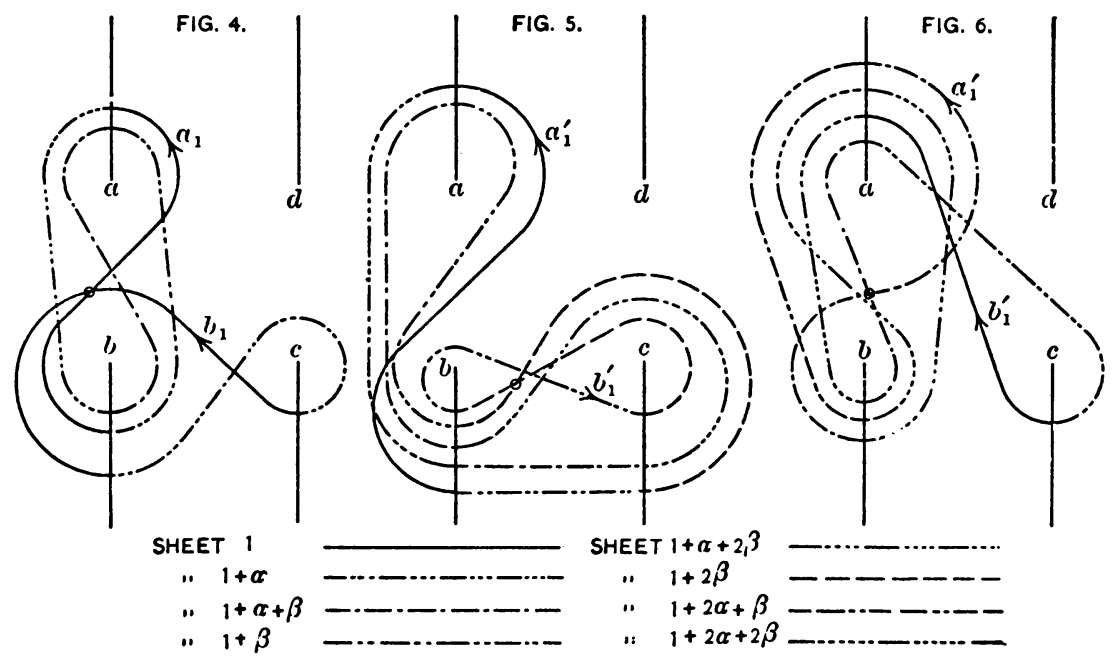


Using the same notation as in the preceding case the values of the integral along the paths in the figure take the form

$$
A_{1}=\alpha_{1}+\beta_{1+a}-\alpha_{1+\beta}-\beta_{1} \text { and } \quad B_{1}=\beta_{1}-\gamma_{1} \text {. }
$$

In determining the effect on the moduli of a monodromy of the branch-points, we first interchange $b$ and $c$ by a positive rotation. The paths $a_{1}$ and $b_{1}$ are transformed at the same time into those given in Fig. 5 . The values of the integral along these new paths are

$$
A_{1}^{\prime}=A_{1}+\left(\sigma^{-\beta}-\sigma^{-\alpha-\beta}\right) B_{1} \quad \text { and } \quad B_{1}^{\prime}=-\sigma^{-\beta} B_{1} .
$$

Dividing as in the previous class, we get

$$
\omega_{1}^{\prime}=-\sigma^{\beta} \omega_{1}+\sigma^{-\alpha}-1,
$$

which substitution is denoted by $S_{1}$.

In the next place rotate $a$ about $b$ positively. The paths $a_{1}$ and $b_{1}$ become those of Fig. 6. Integration along these new paths gives

$$
A_{1}^{\prime}=\sigma^{-(a+\beta)} A_{1}=A_{1}(\text { since } \alpha+\beta=\nu) \quad \text { and } \quad B_{1}^{\prime}=A_{1}+B_{1} \text {. }
$$

Dividing, there is obtained

$$
\omega_{1}^{\prime}=\frac{\omega_{1}}{\omega_{1}+1}
$$

which substitution will be denoted by $V_{1}$.

$$
\text { Class 4. } \beta=\gamma, \alpha \neq \beta \neq \delta \text {. }
$$

The diagram for this case is the same as that given in Fig. 4. The values of the integral along the paths in the figure are as before,

$$
A_{1}=\alpha_{1}+\beta_{1+\alpha}-\alpha_{1+\beta}-\beta_{1} \text { and } B_{1}=\beta_{1}-\gamma_{1} \text {. }
$$

Interchanging $b$ and $c$, the new paths are shown in Fig. 5. Integration along these paths gives,

$$
A_{1}^{\prime}=A_{1} \div\left(\sigma^{-\beta}-\sigma^{-a-\beta}\right) B_{1} \quad \text { and } \quad B_{1}^{\prime}==-\sigma^{-\beta} B_{1} .
$$

Thence, there is obtainea which will be denoted by $S_{1}$.

$$
\omega_{i}^{\prime}=-\sigma^{\beta} \omega_{1}+\sigma^{-\alpha}-1
$$

By the rotation of $a$ about $b$, the transformed paths become those of Fig. 6 . The new integrals are then

whence

$$
A_{1}^{\prime}=\sigma^{-(a+\beta)} A_{1} \quad \text { and } \quad B_{1}^{\prime}=A_{1}+B_{1}
$$

which we call $V_{1}$.

$$
\omega_{1}^{\prime}=\frac{\sigma^{-(\alpha) \beta)} \omega_{1}}{\omega_{1}+1}
$$


Class 5. $\alpha \neq \beta \neq \gamma \neq \delta$.

The diagram for this class is given in Fig. 7 .

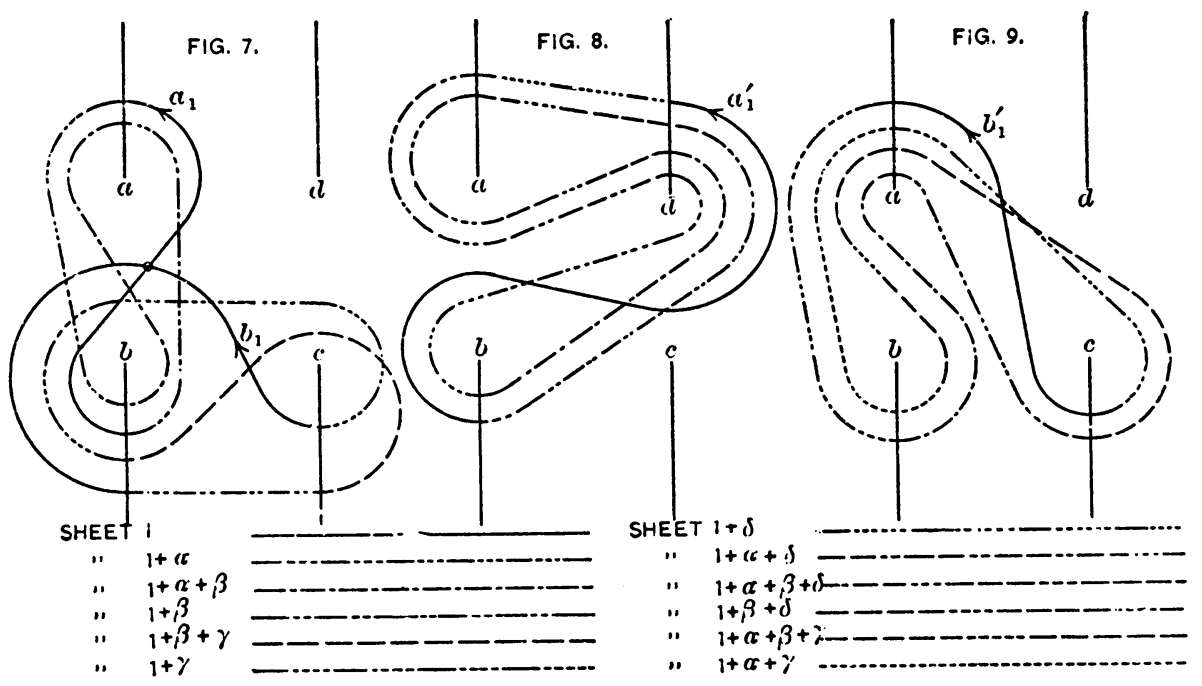

By the rotation of $a$ about $d$ positively, the $a_{1}$ path is transformed into that shown in Fig. 8, the $b_{1}$ path being unaltered. The integral along the $a_{1}^{\prime}$ path then is

whence we get

$$
A_{1}^{\prime}=\sigma^{\beta+\gamma} A_{1}+\left(\sigma^{\gamma}-\sigma^{-a+\gamma}\right) B_{1},
$$

which is denoted by $S_{1}$.

$$
\omega_{1}^{\prime}=\sigma^{\beta+\gamma} \omega_{1}+\sigma^{\gamma}-0^{-a+\gamma},
$$

Again, by the rotation of $a$ about $l$, the $a_{1}$ path is transformed into the $a_{1}^{\prime}$ path of Fig. 6, and the $b_{1}$ path into that of Fig. 9. From this is obtained

$$
A_{1}^{\prime}=\sigma^{-(a+\beta)} A_{1} \quad \text { and } \quad B_{1}^{\prime}=\left(1-\sigma^{-\gamma}\right) A_{1}+B_{1} .
$$

Diviling $A_{1}^{\prime}$ by $B_{1}^{\prime}$ gives

$$
\omega_{1}^{\prime}=\frac{\sigma^{-(a+\beta)} \omega_{1}}{\left(1-\sigma^{-\gamma}\right) \omega_{1}+1} .
$$

This substitution is denoted by $V_{1}$.

\section{\$3. Linear relations among the purcumeters $\omega_{i}$.}

We next investigate under what conditions any given $\omega$ is linearly connected with other $\omega$ 's and with how many others. The following is the method of procedure. When any two branch-points are interchanged, or when the line joining any two is rotated positively about its middle point until the branchpoints resume their original position, a linear substitution on $\omega_{1}$ is obtained, as 
was seen in $\S 2$, while simultaneous transformations are produced on all the other $\omega$ 's. For each value of $q$ there corresponds one $\omega$, or two $\omega$ 's in case $\alpha^{\prime}+\beta^{\prime}+\gamma^{\prime}+\delta^{\prime}=3 \nu$ (see $\S 1$ ), and the transformation for $\omega_{i}$ will be obtained from that for $\omega_{1}$ by replacing $\sigma$ by $\sigma^{q}$ and writing $\omega_{i}$ for $\omega_{1}$.

If a linear relation between $\omega_{i}$ and $\omega_{1}$ is possible, let it be assumed in the form

$$
\omega_{1}=\frac{a \omega_{i}+b}{c \omega_{i}+d}
$$

To determine $a, b, c, d$ the relation $(A)$ is substituted in the transformations $S_{1}$ and $V_{1}$ for the several classes of $\S 2$, which, after solving for $\omega_{i}^{\prime}$, gives transformations $S_{i}$ and $V_{i}$ on $\omega_{i}$. Next, assume that the substitutions derived by these two different processes are identical. Comparing the coefficients of $\omega_{i}$, we obtain three equations from each of the transformations $S_{i}$ and $V_{i}$, from which to determine the values of $a, b, c, d$. In general, these conditions are satisfied only by $q=\nu-1$. But for particular values of $\alpha, \beta, \gamma, \delta$ the number of different values for $q$ satisfying them may be at most three for class 4, and at most seven for class 5 . The following is the application of the method.

$$
\text { Class 2. } \alpha=\beta=\gamma \neq \delta \text {. }
$$

The substitution $S_{1}$ or $\omega_{1}^{\prime}=-\sigma^{a} \omega_{1}-1$ becomes, by replacing $\sigma$ by $\sigma^{q}$ and changing $\omega_{1}$ into $\omega_{i}, S_{i}$ or $\omega_{i}^{\prime}=-\sigma^{q a} \omega_{i}-1$. Substituting $(A)$ in $S_{1}$ and solving for $\omega_{i}^{\prime}$, we get

$$
\omega_{i}^{\prime}=\frac{\omega_{i}\left[-a d \sigma^{\alpha}-c d-b c\right]-b d \sigma^{\alpha}-d^{2}-b d}{\omega_{i}\left[a c+a \imath \sigma^{\alpha}+c^{2}\right]+a d-b c \sigma^{\alpha}+c d} .
$$

Comparison of the coefficients gives the equations

The substitution

$$
\begin{aligned}
& \frac{a d \sigma^{a}+c d+b c}{a d+b c \sigma^{a}+c d}=\sigma^{a q} ; \\
& \frac{b d \sigma^{a}+d^{2}+b d}{a d+b c \sigma^{a}+c d}=1 ; \\
& a c+a c \sigma^{a}+c^{2}=0 .
\end{aligned}
$$

$$
V_{1} \quad \text { or } \quad \omega_{1}^{\prime}=\frac{-\sigma^{-a} \omega_{1}}{\omega_{1}+1}
$$

on changing $\sigma$ into $\sigma^{q}$ and $\omega_{1}$ into $\omega_{i}$, becomes

$$
V_{i} \quad \text { or } \quad \omega_{i}^{\prime}=\frac{-\sigma^{-a q} \omega_{i}}{\omega_{i}+1}
$$

Trans. Am. Math. Soc. $\$ 9$ 
Using relation $(A)$ and solving for $\omega_{i}^{\prime}$, we get

$$
\omega_{i}^{\prime}=\frac{\omega_{i}\left[-a d \sigma^{-a}-a b-b c\right]-b d \sigma^{-a}-b d-b^{2}}{\omega_{i}\left[a^{2}+a c+a c \sigma^{-a}\right]+a b+a d+b c \sigma^{-a}} .
$$

Comparison of coefficients gives the equations,

$$
\begin{aligned}
& a d \sigma^{-a}+a b+b c \\
& a b+a d+b c \sigma^{-a}=\sigma^{-a q} ; \\
& b d \sigma^{-a}+b d+b^{2}=0 ; \\
& a^{2}+a c+a c \sigma^{-a} \\
& a b+a d+b c \sigma^{-a}=1
\end{aligned}
$$

Theie are two values of $c$ in terms of $a$ from equation (3), and two values of $b$ in terms of $d$ from equation (5), giving four different solutions of cases to be examined, which are,

$$
\begin{array}{rlrl}
\text { Case } 1, c=-a\left(1+\sigma^{a}\right), & =-d\left(1+\sigma^{-a}\right) ; \\
\text { “ } 2, c=-a\left(1+\sigma^{a}\right), b & =0 ; \\
\text { “ } 3, c=0, & b & =-d\left(1+\sigma^{-a}\right) ; \\
\text { “ } 4, c=0, & b & =0 .
\end{array}
$$

If each of these pairs of solutions is used in connection with equations (2) or (6) as the case may be, it is possible to express $b, c, d$ in terms of $a$. The above four different solutions then become

$$
\begin{aligned}
& \text { Case 1, } c=-a\left(1+\sigma^{a}\right), b=a\left(1+\sigma^{a}\right), d=-u \sigma^{a} \text {; } \\
& \text { " } 2, c=-a\left(1+\sigma^{a}\right), b=0, \quad d=-a \sigma^{a} \text {; } \\
& \text { “ } 3, c=0, \quad b=a\left(1+\sigma^{\alpha}\right), d=-a \sigma^{\alpha} \text {; } \\
& \text { “ } 4, c=0, \quad b=0, \quad d=a \text {. }
\end{aligned}
$$

Solutions 2 and 3 are to be discarded since equation (6) is not satisfied by these values unless $\alpha=\nu / 2$. From $\alpha+\beta+\gamma+\delta=2 \nu$ it then follows that $\delta$ must also equal $\nu / 2$, which is impossible for this class.

If the values from solution 4 are substituted in equations (1) and (4), we get $\sigma^{\alpha q}=\sigma^{\alpha}$ and $\sigma^{-\alpha q}=\sigma^{-\alpha}$, whence is deduced the congruence $(q-1) \alpha \equiv 0(\bmod . \nu)$. Since $\alpha$ and $\nu$ must be relatively prime, the only value of $q$ which satisfies this congruence is unity, and $\omega_{i}$ is then identical with $\omega_{1}$.

Substituting the values from solution 1 in equations (1) and (4), we obtain the congruence $(q+1) \alpha \equiv 0(\bmod . \nu)$. The only value of $q$ less than $\nu$ satisfying this is $q=\nu-1$.

Since any one of the $p^{\prime} \omega$ 's may be taken for $\omega_{1}$, we readily deduce the result; 
Theorem II. For Class 2, any given w, corresponding to a value $q_{1}$ of $q$, can be linearly connected with but one other $\omega$, namely with that one corresponding to a value $q_{2}$ of $q$ satisfying the relation $q_{1}+q_{2}=\nu$.

If $2 n=p^{\prime}$ denotes the number of $\omega^{\prime}$ s, then there are at least $n$ which are linearly independent.

$$
\text { Class 3. } \beta=\gamma, \alpha=\delta, \alpha \neq \beta \text {. }
$$

The transformation

becomes

$$
S_{1} \quad \text { or } \quad \omega_{1}^{\prime}=-\sigma^{\beta} \omega_{1}+\sigma^{-a}-1
$$

$$
S_{i} \quad \text { or } \quad \omega_{i}^{\prime}=-\sigma^{q \beta} \omega_{i}+\sigma^{-q a}-1 \text {. }
$$

Substituting relation $(A)$ in $S_{1}$, and solving for $\omega_{i}^{\prime}$, as before, we find

$$
\omega_{i}^{\prime}=\frac{\omega_{i}\left[d c \sigma^{-a}-d c-a d \sigma^{\beta}-b c\right]+d^{2} \sigma^{-a}-d^{2}-b d \sigma^{\beta}-b d}{\omega_{i}\left[a c+c^{2}+a c \sigma^{\beta}-c^{2} \sigma^{-a}\right]+a d+c d+h c \sigma^{\beta}-c d \sigma^{-a}} .
$$

Comparing coefficients, we have the equations,

$$
\begin{gathered}
\frac{d c \sigma^{-a}-d c-a d \sigma^{\beta}-b c}{a d+c d+b c \sigma^{\beta}-c d \sigma^{-a}}=-\sigma^{\imath \beta} ; \\
\frac{d^{2} \sigma^{-a}-d^{2}-b d \sigma^{\beta}-b d}{a d+c d+b c \sigma^{\beta}-c d \sigma^{-a}}=-1+\sigma^{-q a} \\
a c+c^{2}+a c \sigma^{\beta}-c^{2} \sigma^{-a}=0 .
\end{gathered}
$$

The transformation

becomes

$$
V_{1} \text { or } \omega_{1}^{\prime}=\frac{\omega_{1}}{\omega_{1}+1}
$$

$$
V_{i} \text { or } \omega_{i}^{\prime}=\frac{\omega_{i}}{\omega_{i}+1} \text {. }
$$

Relation $(A)$, applied to substitution $V_{1}$, gives

$$
\omega_{i}^{\prime}=\frac{\omega_{i}[a d-a b-b c]-b^{2}}{\omega_{i} a^{2}+a b+a d-b c} .
$$

Equations (4), (5), (6) follow from the comparison of coefficients :

$$
\begin{aligned}
\frac{a d-a b-b c}{a d+a b-b c} & =1 ; \\
-b^{2} & =0 ; \\
\frac{a^{2}}{a d+a b-b c} & =1 .
\end{aligned}
$$


From equation (5), $b=0$ and from (3) we obtain $c=0$, or $c=a\left(1+\sigma^{\beta}\right) /\left(\sigma^{-x}-1\right)$. By the use of $c=0$, equation (6) gives $d=a$, and equations (1) and (2) give $\sigma^{q \beta}=\sigma^{\beta}$ and $\sigma^{q a}=\sigma^{\alpha}$, whence $(q-1) \beta \equiv 0(\bmod . \nu)$ and $(q-1) \alpha \equiv 0($ mod. $\nu)$. The only value of $q<\nu$ satisfying these congruences is $q=1$, since $x$ and $\beta$ must be relatively prime, otherwise the Riemann surface would reduce.

Taking $c=a\left(1+\sigma^{\beta}\right) /\left(\sigma^{-\alpha}-1\right)$ we get, from equation (6), $d=a$, and from equations $(1)$ and $(2) \sigma^{q \beta}=\sigma^{-\beta}$ and $\sigma^{-q a}=\sigma^{a}$, whence $(q+1) \beta \equiv 0(\bmod . \nu)$ and $(q+1) \alpha \equiv 0$ (mod. $\nu)$. It is evident that $q=\nu-1$ is the only value of $q<\nu$ that satisfies these last congruences, since $\alpha$ and $\beta$ must be relatively prime.

Accordingly the result for Class 3 is the same as for Class 2 . In particular there are at least $n=p^{\prime} / 2$ linearly independent $\omega$ 's.

$$
\text { Class 4. } \beta=\gamma, \alpha \neq \beta \neq \delta \text {. }
$$

The transformation

becomes

$$
S_{1} \text { or } \omega_{1}^{\prime}=-\sigma^{\beta} \omega_{1}-1+\sigma^{-a}
$$

$$
S_{i} \quad \text { or } \quad \omega_{i}^{\prime}=-\sigma^{4 \beta} \omega_{i}-1+\sigma^{-q a} .
$$

Using relation $(A)$ in $S_{1}$ and solving for $\omega_{i}^{\prime}$, we obtain

$$
\omega_{i}^{\prime}=\frac{\omega_{i}\left[c \dot{d}\left(\sigma^{-a}-1\right)-a d \sigma^{\beta}-b c\right]+d^{2}\left(\sigma^{-a}-1\right)-b d \sigma^{\beta}-b d}{\omega_{i}\left[a c+a c \sigma^{\beta}-c^{2}\left(\sigma^{-a}-1\right)\right]+a d+b c \sigma^{\beta}-c d\left(\sigma^{-a}-1\right)} .
$$

Comparison as before gives the equations,

$$
\begin{aligned}
& \frac{c d\left(\sigma^{-a}-1\right)-a d \sigma^{\beta}-b c}{a d+b c \sigma^{\beta}-c d\left(\sigma^{-a}-1\right)}=\sigma^{q \beta} ; \\
& \frac{d^{2}\left(\sigma^{-a}-1\right)-b d \sigma^{\beta}-b d}{a d+b c \sigma^{\beta}-c d\left(\sigma^{-\alpha}-1\right)}=\sigma^{-q a}-1 ; \\
& a c+a c \sigma^{\beta}-c^{2}\left(\sigma^{-\alpha}-1\right)=0 .
\end{aligned}
$$

The transformation

becomes

$$
V_{1} \quad \text { or } \quad \omega_{1}^{\prime}=\frac{\sigma^{-(a+\beta)} \omega_{1}}{\omega_{1}+1}
$$

$$
V_{i} \quad \text { or } \quad \omega_{i}^{\prime}=\frac{\sigma^{-(a+\beta) q} \omega_{i}}{\omega_{i}+1}
$$

Then using relation $(A)$ in $V_{1}$ and solving for $\omega_{i}^{\prime}$, we get

$$
\omega_{i}^{\prime}=\frac{\omega_{i}\left[a d \sigma^{-(a+\beta)}-a b-b c\right]+b d\left[\sigma^{-(a+\beta)}-1\right]-b^{2}}{\omega_{i}\left[a^{2}+a c\left(1-\sigma^{-a-\beta}\right)\right]+a d+a b-b c \sigma^{-(a+\beta)}},
$$


whence we obtain the equations,

$$
\begin{gathered}
\frac{a d \sigma^{-(a+\beta)}-a b-b c}{a d+a b-b c \sigma^{-(a+\beta)}}=\sigma^{-(a+\beta) q} ; \\
b d \sigma^{-(a+\beta)}-b^{2}-b d=0 ; \\
\frac{a^{2}+a c\left[1-\sigma^{-(a+\beta)}\right]}{a d+a b-b c \sigma^{-(a+\beta)}}=1 .
\end{gathered}
$$

Equations (3) and (5) give two values of $c$ in terms of $a$, and two values of $b$ in terms of $d$, respectively, making four different solutions to be examined; these are

$$
\begin{array}{lll}
1, & c=a \frac{1+\sigma^{\beta}}{\sigma^{-a}-1}, & b=d\left[\sigma^{-(a+\beta)}-1\right] ; \\
2, & c=0, & b=d\left[\sigma^{-(a+\beta)}-1\right] ; \\
3, & c=0, & b=0 ; \\
4, & c=a \frac{1+\sigma^{\beta}}{\sigma^{-a}-1}, & b=0 .
\end{array}
$$

These pairs of values, each combined with equation (6), enable us to express $\delta$, $c$, and $d$ in terms of $a$, which values of $b, c, d$ substituted in (1), (2), and (4) give the following conditions, only twc of which are independent.

For solution 1, we have

$$
\sigma^{q \beta}=\sigma^{-\beta}, \quad \sigma^{-q a}=\sigma^{a} \quad \text { and } \quad \sigma^{-(a+\beta) q}=\sigma^{a+\beta},
$$

whence it follows that $(q+1) \alpha \equiv(q+1) \beta \equiv 0(\bmod . \nu)$.

For solution 2,

$$
\sigma^{\alpha \beta}=\sigma^{\beta}, \quad \sigma^{-q a}=\sigma^{\alpha+2 \beta} \quad \text { and } \quad \sigma^{-(a+\beta) q}=\sigma^{a+\beta},
$$

whence $(q-1) \beta \equiv(q+1)(\alpha+\beta) \equiv 0(\bmod . \nu)$.

For solution 3,

$$
\sigma^{q \beta}=\sigma^{-\beta}, \quad \sigma^{-q a}=\sigma^{-\alpha}, \quad \text { and } \quad \sigma^{-(a+\beta) q}=\sigma^{-(a+\beta)},
$$

whence $(q-1) \beta \equiv(q-1) \alpha \equiv 0(\bmod . \nu)$.

For solution 4,

$$
\sigma^{q \beta}=\sigma^{-\beta}, \quad \sigma^{-q a}=\sigma^{-(a+\xi \beta)} \quad \text { and } \quad \sigma^{-(a+\beta) g}=\sigma^{-(a+\beta)},
$$

whence $(q+1) \beta \equiv(q-1)(\alpha+\beta) \equiv 0(\bmod \nu)$.

It can be easily shown that $q=\nu-1$ is the only value of $q<\nu$ that will satisfy the conditions of solution 1 , and $q=1$ the only value that will satisfy 
the conditions of solution 3 , for otherwise a factor of $\nu$ would be common to $\alpha$ and $\beta$, and the Riemann surface would be reducible.

Since $\alpha, \beta, \nu$ cannot have a common factor, and neither can $(\alpha+\beta), \beta, \nu$, we have in solution 2 to consider only the following possibilities :

First, $\beta$ and $\nu$ may be relatively prime ;

Second, $(\alpha+\beta)$ and $\nu$ may be relatively prime;

Third, $\beta$ and $\nu$ may have a common factor, and at the same time $(\alpha+\beta)$ and $\nu$ may have a common factor.

First. If $\beta$ and $\nu$ are relatively prime, we find from $(q-1) \beta \equiv 0(\bmod . \nu)$ that $q=1$, which excludes this possibility, since $q$ must be different from unity.

Second. If $(\alpha+\beta)$ and $\nu$ are relatively prime it is found from $(q+1)(\alpha+\beta) \equiv 0$ $(\bmod . \nu)$ that $q=\nu-1$. But this will not satisfy $(q-1) \beta \equiv 0(\bmod . \nu)$ unless $\beta=\nu / 2$. This value of $\beta$ makes solution 2 coincide with solution 1 .

Third. Suppose $\alpha+\beta=\alpha_{1} \alpha_{2}, \beta=\beta_{1} \beta_{2}, \nu=\alpha_{1} \beta_{1} \nu_{1}$, in which $\alpha_{1}$ and $\beta_{1}$ are of necessity relatively prime. Then $(q-1) \beta=(q-1) \beta_{1} \beta_{2}=p_{1} x_{1} \beta_{1} \nu_{1}$, say, whence $(q-1) \beta_{2}=p_{1} \alpha_{1} \nu_{1}$. But $\beta_{2}, \alpha_{1}$, and $\nu_{1}$ are relatively prime. Put $p_{1}=\beta_{2} p_{1}^{\prime}$, then $q-1=p_{1}^{\prime} \alpha_{1} \nu_{1}$. Similarly, from $(q+1)(\alpha+\beta)=p_{2} \nu$ we find $q+1=p_{2}^{\prime} \beta_{1} \nu_{1}$, if $p_{2}=p_{2}^{\prime} \alpha_{2}$. Hence $q-1$ and $q+1$ must contain $\nu_{1}$ as a factor. But, since $q-1$ and $q+1$ differ only by $2, \nu_{1}$ can have only the values 1 and 2. There is but one value of $q$ between 1 and $\nu$ which will satisfy the equations $q-1=p_{1}^{\prime} \alpha_{1} \nu_{1}$, and $q+1=p_{2}^{\prime} \beta_{1} \nu_{1}$. For, suppose there are two such values, which denote by $q_{1}$ and $q_{2}$ where $q_{2}>q_{1}$. Substituting these values in the equations, we have

$$
\begin{aligned}
& q_{1}-1=m_{1} \alpha_{1} \nu_{1}, \quad \text { and } \quad q_{2}-1=m_{2} x_{1} \nu_{1} . \\
& q_{1}+1=n_{1} \beta_{1} \nu_{1}, \quad \text { and } \quad q_{2}+1=n_{2} \beta_{1} \nu_{1} \text {, }
\end{aligned}
$$

in which $m_{1}, m_{2}, n_{1}, n_{2}$ denote the corresponding values of $p_{1}^{\prime}$ and $p_{2}^{\prime}$. From these equations, we derive $q_{2}-q_{1}=\left(m_{2}-m_{1}\right) \alpha_{1} \nu_{1}=\left(n_{2}-n_{1}\right) \beta_{1} \nu_{1}$, which shows that $\left(q_{2}-q_{1}\right)$ contains $\alpha_{1}, \beta_{1}$, and $\nu_{1}$ as factors, and since these are relatively prime, $\left(q_{2}-q_{1}\right)$ must equal $p x_{1} \beta_{1} \nu_{1}$ or $p \nu$. Therefore $q_{2}=p \nu+q_{1}$, whence it follows that $q_{2}>\nu$.

For solution 4, the same restrictions and possibilities apply as were employed in solution 2, giving the same results.

Summarizing, and observing that the same line of argument would hold if we started with any other $\omega$ in place of $\omega_{1}$, we note that in solution 1 , any given $\omega$ can be linearly connected with at most one other. The corresponding values of $q$, say $q_{1}$ and $q_{2}$, are connected by the relation $q_{1}+q_{2}=\nu$. In solution 2 , the given $\omega$ may be connected with but one other, possibly a different one from that of solution 1 ; in solution 3 , relation $(A)$ becomes identity; and in solution 4 , there may possibly be a still different $\omega$ connected with this given one. Hence the result : 
Theorex III. For Class 4, any given $\omega$ may be linearly connected with not more than three others.

If it should happen for a particular Riemann surface of class 4 that each $\omega$ is linearly connected with three others, then the number of the $2 n$ which are linearly independent would be $2 n / 4$ or $n / 2$. Accordingly, if the number of $\omega$ 's is greater than four, they are not all linearly expressible in terms of one.

The transformation

$$
\text { Class 5. } \alpha \neq \beta \neq \gamma \neq \delta .
$$

becomes

$$
S_{1} \quad \text { or } \quad \omega_{1}^{\prime}=\sigma^{(\beta+\gamma)} \omega_{1}+\sigma^{\gamma}-\sigma^{\gamma-a}
$$

$$
S_{i} \quad \text { or } \quad \omega_{i}^{\prime}=\sigma^{(\beta+\gamma)} \omega_{i}+\sigma^{q \gamma}-\sigma^{\prime / \gamma-\alpha)} \text {. }
$$

Substituting relation $(A)$ in $S_{1}$ and solving for $\omega_{i}^{\prime}$ we obtain

$$
\omega_{i}^{\prime}=\frac{\omega_{i}\left[a d \sigma^{\beta+\gamma}+c d\left(\sigma^{\gamma}-\sigma^{\gamma-a}\right)-b c\right]+b d \sigma^{\beta+\gamma}+d^{2}\left(\sigma^{\gamma}-\sigma^{\gamma-a}\right)-b d}{\omega_{i}\left[a c-a c \sigma^{\beta}+\gamma-c^{2}\left(\sigma^{\gamma}-\sigma^{\gamma-a}\right)\right]+a d-b c \sigma^{\beta+\gamma}-c d\left(\sigma^{\gamma}-\sigma^{\gamma-\alpha}\right)} .
$$

As before, we derive the equations,

$$
\begin{gathered}
\frac{a d \sigma^{\beta+\gamma}+c d\left[\sigma^{\gamma}-\sigma^{\gamma-a}\right]-b c}{a d-c d\left[\sigma^{\gamma}-\sigma^{\gamma-a}\right]-b c \sigma^{\beta+\gamma}}=\sigma^{(\beta+\gamma ; q} ; \\
\frac{b d\left(\sigma^{\beta+\gamma}-1\right)+d^{2}\left[\sigma^{\gamma}-\sigma^{\gamma-a}\right]}{a d-c d\left[\sigma^{\gamma}-\sigma^{\gamma-a}\right]-b c \sigma^{\beta+\gamma}}=\sigma^{q \gamma}-\sigma^{g(\gamma-a)} ; \\
a c\left(1-\sigma^{\beta+\gamma}\right)-c^{2}\left(\sigma^{\gamma}-\sigma^{\gamma-a}\right)=0 .
\end{gathered}
$$

The transformation

becomes

$$
V_{1} \quad \text { or } \quad \omega_{1}^{\prime}=\frac{\sigma^{-(a+\beta)} \omega_{1}}{\left(1-\sigma^{-\gamma}\right) \omega_{1}+1}
$$

$$
V_{i} \quad \text { or } \quad \omega_{i}^{\prime}=\frac{\sigma^{-(a+\beta) /} \omega_{i}}{\left(1-\sigma^{-q \gamma}\right) \omega_{i}+1}
$$

Again, by substituting and solving, we have

$$
\omega_{i}^{\prime}=\frac{\omega_{i}\left[a d \sigma^{-(a+\beta)}-b c-a b\left(1-\sigma^{-\gamma}\right)\right]+b d \sigma^{-(a+\beta)}-b d-b^{2}\left(1-\sigma^{-\gamma}\right)}{\omega_{i}\left[a^{2}\left(1-\sigma^{-\gamma}\right)+a c-a c \sigma^{-(a+\beta)}\right]+a b\left(1-\sigma^{-\gamma}\right)+a d-b c \sigma^{-(a+\beta}} .
$$

Comparison of coefficients gives the remaining equations,

$$
\begin{gathered}
a d \sigma^{-(a+\beta)}-b c-a b\left(1-\sigma^{-\gamma}\right) \\
a b\left(1-\sigma^{-\gamma}\right)+a d-b c \sigma^{-(a+\beta)}=\sigma^{-(a+\beta) q} ; \\
b c l \sigma^{-(a+\beta)}-b d-b^{2}\left(1-\sigma^{-\gamma}\right)=0 ; \\
a^{2}\left(1-\sigma^{-\gamma}\right)+a c-a c \sigma^{-(a+\beta)} \\
a b\left(1-\sigma^{-\gamma}\right)+a d-b c \sigma^{-(u+\beta)}=1-\sigma^{-\gamma \gamma} .
\end{gathered}
$$


The third and fifth equations give two values of $c$ in terms of $a$ and two for $b$ in terms of $d$ respectively, making four cases to be examined, namely,

Case 1.

“ 2.

“ 3 .

“4.

$$
c=\frac{a\left(1-\sigma^{\beta+\gamma}\right)}{\sigma^{\gamma}-\sigma^{\gamma-a}}, \quad z=d \frac{\sigma^{-(a+\beta)}-1}{1-\sigma^{-\gamma}} ;
$$

$$
b=0 \text {; }
$$$$
l=d \frac{\sigma^{-(a+\beta)}-1}{1-\sigma^{-\gamma}}
$$

$$
c=0,
$$$$
b=0 \text {. }
$$

These pairs of values combined with the preceding equations give the following conditions :

Case 1. (a) $\sigma^{\alpha(\beta+\gamma)}=\sigma^{-(\beta+\gamma)}$;

(b) $\sigma^{\text {ra+ }+\beta)}=\sigma^{-(a+\beta)}$;

(c) $\sigma^{\alpha \gamma}-\sigma^{\gamma \gamma-a)}+\sigma^{-q a}=\sigma^{a}-\sigma^{-(\gamma-a)}+\sigma^{-\gamma}$.

From $(a)$ and $(\tilde{b}), \sigma^{q(y-a)}=\sigma^{-(\gamma-\imath)}$, whence $\sigma^{q \gamma}=\sigma^{-(\gamma-a)+q a}$.

Substitute these in $(c)$, factor, and get the equation

$$
\left[\sigma^{(q+1) a}-1\right]\left[\sigma^{-\gamma}-\sigma^{q a}\right]=0 .
$$

Case 2. (a) $\sigma^{q(\beta+\gamma)}=\sigma^{\beta+\gamma}$;

(b) $\sigma^{-(a+\beta) q}=\sigma^{-(a+\beta)}$;

(c) $\sigma^{\gamma \gamma}+\sigma^{-q u}-\sigma^{q(\gamma-a)}=\sigma^{-a}-\sigma^{\gamma-a}+\sigma^{\gamma}$.

Equations $(a)$ and $(b)$ give $\sigma^{\lambda(\gamma-a)}=\sigma^{\gamma-a}$, whence $\sigma^{q \gamma}=\sigma^{\gamma-a+q a}$.

Substituting these in $(c)$ and factoring we obtain

$$
\left[\sigma^{(q-1) a}-1\right]\left[\sigma^{-\varsigma a}-\sigma^{\gamma}\right]=0 .
$$

Case 3. (a) $\sigma^{\prime(\beta+\gamma)}=\sigma^{\beta+\gamma}$;

(b) $\sigma^{-(a+\beta) \eta}=\sigma^{\alpha+\beta}$.

(c) $\sigma^{2 \gamma}-\sigma^{\prime(\gamma-a,}+\sigma^{-q a}=\sigma^{\beta}+\sigma^{a+\beta+\gamma}-\sigma^{a+2 \beta+\gamma}$.

Then $(a)$ and $(b)$ give $\sigma^{q(\gamma-a)}=\sigma^{a+2 \beta+\gamma}$, whence $\sigma^{q \gamma}=\sigma^{a+2 \beta+\gamma+z a}$.

Substituting these in $(c)$ and factoring, we obtain,

$$
\left[\sigma^{q a+\beta}-1\right]\left[\sigma^{a+\beta+\gamma}-\sigma^{-q a}\right]=0 .
$$


Case 4. (a) $\sigma^{g(\beta+\gamma)}=\sigma^{-(\beta+\gamma)}$;

(b) $\sigma^{-(a+\beta) q}=\sigma^{-(a+\beta)}$;

(c) $\sigma^{\alpha \gamma}+\sigma^{-q a}-\sigma^{\alpha \gamma-a)}=\sigma^{-\beta}+\sigma^{-(a+\beta+\gamma}-\sigma^{-(a+2 \beta+\gamma)}$.

Using $(a)$ and $(b), \sigma^{q(\gamma-a)}=\sigma^{-(a+2 \beta+\gamma)}$, whence $\sigma^{q \gamma}=\sigma^{-(a+2 \beta+\gamma)+q a}$. Finally, putting these in $(c)$ and factoring, we have

$$
\left[\sigma^{q a-\beta}-1\right]\left[\sigma^{-(a+\beta+\gamma)}-\sigma^{-q a}\right]=0 .
$$

For case 1 , if $\sigma^{(q+1) a}=1$, we find from $(a)$ and $(b)$ that $(q+1) \alpha$, $(q+1)(\beta+\gamma)$, and $(q+1)(\alpha+\beta)$ are all divisible by $\nu$ and hence by every factor of $\nu$. If $\alpha, \alpha+\beta$, and $\beta+\gamma$ should all contain a factor $\nu_{1}$ of $\nu$, then $\beta, \gamma, \delta$ would also contain $\nu_{1}$, and the equation of the Riemann surface would reduce. Hence $q+1$ must be divisible by all the factors of $\nu$, that is $q+1=\nu$, or $q=\nu-1$.

Again, if $\sigma^{g a-\gamma}-1=0$, equations $(a)$ and $(b)$ give

$$
q \alpha-\gamma \equiv(q+1)(\beta+\gamma) \equiv(q+1)(\alpha+\beta) \equiv 0(\bmod . \nu) .
$$

For these congruences, there are only two possibilities, namely,

and

$$
\alpha+\beta=\gamma_{1} \gamma_{2}, \quad \beta+\gamma=\alpha_{1} \alpha_{2}, \quad \nu=x_{1} \gamma_{1} \nu_{1},
$$

$$
\alpha+\beta=\gamma_{1} \nu_{1}, \quad \beta+\gamma=\alpha_{1} \nu_{1}, \quad \nu=\nu_{1} \nu_{2} .
$$

For the first possibility, we find $q=\nu-1$, which value of $q$ satisfies all three congruences only when $\alpha+\gamma=\nu$. For the second possibility, we can show, by a method analogous to that used in the third possibility of class 4 , that there is but one value of $q$ less than $\nu$ and different from unity that satisfies all three congruences.

There are accordingly not more than two different values of $q<\nu$ (one of which is $\nu-1$ ) which will satisfy the conditions of case 1.

For case 2 , if $\sigma^{(q-1) a}-1=0$, we get from $(a)$ and $(b)$,

$$
(q-1) \alpha \equiv(q-1)(\alpha+\beta) \equiv(q-1)(\beta+\gamma) \equiv 0(\bmod . \nu) .
$$

The same possibilities, and only those, apply here as in the first part of case 1 , whence $q=1$.

Again, if $\sigma^{q a-\gamma}-1=0$, we have the congruences

$$
q \alpha+\gamma \equiv(q-1)(\alpha+\beta) \equiv(q-1)(\beta+\gamma) \equiv 0(\bmod . \nu) .
$$

There are here also only two possibilities, as in the second part of case 1 . Consideration of these shows that $q$ can have not more than one value less than $\nu$.

Thus there is not more than one value of $q$ different from unity and less than $\nu$ which satisfies the conditions of case 2 . 
For case 3 , if $\sigma^{\gamma a+\beta}-1=0$, there are the congruences

$$
q \alpha+\beta \equiv(q-1)(\beta+\gamma) \equiv(q+1)(\alpha+\beta) \equiv 0 \quad(\bmod . \nu),
$$

the first of which may have the form $(q-1) \alpha+(\alpha+\beta) \equiv 0(\bmod . \nu)$. In these we have only three possibilities, which are :

First, $(\alpha+\beta)$ and $\nu$ relatively prime;

Second, $(\beta+\gamma)$ and $\nu$ relatively prime;

Third, $\alpha+\beta=\gamma_{1} \gamma_{2}, \beta+\gamma=\alpha_{1} \alpha_{2}, \nu=\gamma_{1} \alpha_{1} \nu_{1}$.

From $(q+1)(\alpha+\beta) \equiv 0$ (mod. $\nu), q$ equals $\nu-1$. This value of $q$ satisfies $(q-1)(\beta+\gamma) \equiv 0(\bmod . \nu)$ only when $\beta+\gamma=\nu / 2, \nu$, or $3 \nu / 2$, and substituted in $q \alpha+\beta \equiv 0(\bmod . \nu)$ gives the congruence $\beta-\alpha \equiv 0(\bmod . \nu)$. But, since $\beta$ and $\alpha$ can not differ by $\nu$, this is impossible unless $\beta=\alpha$. This possibility is therefore to be excluded since it reduces Class 5 to Class 4.

From $(q-1)(\beta+\gamma) \equiv 0$ (mod. $\nu), q$ equals 1 . But this possibility must be excluded since $q$ must be different from unity.

For the third possibility, we can easily show by methods already employed that $q$ can not have more than one value less than $\nu$.

Again, if $\sigma^{2 a+a+\beta+\gamma}-1=0$, we have the congruences

$$
q x+\alpha+\beta+\gamma \equiv(q-1)(\beta+\gamma) \equiv(q+1)(\alpha+\beta) \equiv 0 \quad(\bmod . \nu),
$$

the first of which may have the different forms $(q+1) \alpha+(\beta+\gamma) \equiv q \alpha-\delta \equiv 0$ $(\operatorname{mol} . \nu)$. There are the same possibilities for this second part as for the first. From $(q+1)(\alpha+\beta) \equiv 0(\bmod . \nu)$, for the first possibility, we find $q=\nu-1$, which imposes the condition $\beta+\gamma=\nu$. The second possibility is to be excluded because it reduces Class 5 to Class 4 , since from $(q-1)(\beta+\gamma) \equiv 0$, we deduce $q=1$, which inserted in $q \alpha-\delta \equiv 0$, gives $\alpha=\delta$. For the third possibility we can show by former methods that $q$ can not have more than one value less than $\nu$, which value may be different from those already obtained.

The total result in this case is that there are not more than two distinct values of $q(1<q<\nu)$ possible.

Finally, for case 4 , we have the following two sets of equations,

and

$$
q \alpha-\beta \equiv(q+1)(\beta+\gamma) \equiv(q-1)(\alpha+\beta) \equiv 0 \quad(\bmod . \nu),
$$

$$
(q-1) \alpha-(\beta+\gamma) \equiv(q+1)(\beta+\gamma) \equiv(q-1)(\alpha+\beta) \equiv 0 \quad(\bmod . \nu) .
$$

The relation $(q-1) \alpha-(\beta+\gamma) \equiv 0$ may also be written $q \alpha+\delta \equiv 0$. There are the same possibilities for each of these two sets of equations as in the previous case, for which there are similar conclusions, namely, in case neither $(\beta+\gamma)$ nor $(\alpha+\beta)$ are relatively prime to $\nu$ there are not more than two values of $q(1<q<\nu)$ which satisfy all the conditions. 
Summarizing, we find that in each of the cases 1,3, 4 there may be two distinct values of $q$ different from unity and less than $\nu$, and only one value of $q$ in case 2 , one of these seven values of $q$ being $\nu-1$. Hence,

TheOREM IV. For Class 5, any given $\omega$ cannot be linearly connected with more than seven others.

If it should happen that all the $\omega$ 's under consideration should separate into sets of 8 , those in each set being linearly connected, then the number of those linearly independent would be $2 n / 8$ or $n / 4$. Accordingly, if the number of $\omega$ 's is greater than eight, they are not all linearly expressible in terms of one.

As a result of the investigation of these five classes, we conclude that only a finite number of Riemann surfaces can exist for which all the $\omega$ 's are linearly connected.

\section{PART II.}

In order still further to reduce the finite number of cases which exist, we assume that the automorphic functions under consideration shall be uniform, which requires that the fundamental region for the group of substitutions of any $\omega_{i}$ must be a polygon, the angles at whose vertices are aliquot parts of $2 \pi$. This imposes certain conditions on the generating substitutions of the group. Suppose such a transformation to be written in the form

$$
\frac{\omega^{\prime}-f_{1}}{\omega^{\prime}-f_{2}}=K \frac{\omega-f_{1}}{\omega-f_{2}}
$$

where $f_{1}$ and $f_{2}^{\prime}$ are the two fixed points. Then for the generating substitutions of the groups under consideration, the "multiplier," $K$, must have the form

$$
e^{2 \pi i(p \pm 1 / l)},
$$

in which $p$ and $l(l>1)$ are integers. The method of procedure will be made clear by the consideration of Class $4, \beta=\gamma$.

\section{\$1. Values of $l_{1}, l_{2}$, and $l_{3}$ of Class 4 .}

If the transformations of this class are compounded, there is obtained the transformation $T_{1}=S_{1} V_{1}$, namely,

$$
\omega_{1}^{\prime}=\frac{-\omega_{1}+\sigma^{\alpha}-1}{\omega_{1}+1} .
$$

The value $l_{1}$ of $l$ for this substitution is therefore 2 .

Since the multiplier for the substitution

$$
S_{1} \quad \text { or } \quad \omega_{1}^{\prime}=-\sigma^{\beta} \omega_{1}+\left(\sigma^{-a}-1\right),
$$


is $-\sigma^{\beta}=-e^{2 \pi i \beta / v}$, it follows from the above assumption in regard to $K$, that $\beta$ must satisfy the relation

$$
\frac{\beta}{\nu}+\frac{1}{2}=p \pm \frac{1}{l_{2}}
$$

where $l_{2}$ denotes the values of $l$ for $S_{1}$. From the inequality $0<\beta<\nu$ follow

$$
-\frac{1}{2}<\frac{2 \beta-\nu}{2 \nu}<\frac{1}{2} \quad \text { and } \quad \frac{1}{2}<\frac{2 \beta+\nu}{2 \nu}<\frac{8}{2} .
$$

Hence, since $1 / l_{2}$ is positive and does not exceed $\frac{1}{2}$, formula (1) reduces to

$$
\frac{2 \beta-\nu}{2 \nu}= \pm \frac{1}{l_{2}}
$$

It is evident that $l_{2}$ cannot equal 2 , as otherwise $\beta$ would be divisible by $\nu$. We proceed, then, to investigate the cass $l_{2}>2$. The substitution

$$
S_{i} \quad \text { or } \quad \omega_{i}^{\prime}=-\sigma^{\beta \prime} \omega_{i}+\left(\sigma^{-\alpha^{\prime}}-1\right),
$$

which is simultaneous with $S_{1}$, must also satisfy the same conditions, and hence

$$
\frac{2 \beta^{\prime}-\nu}{2 \nu}= \pm \frac{1}{l_{2}}
$$

from which follows $\beta^{\prime}=k_{2}\left(l_{2} \pm 2\right)$. There are two cases to be considered, according as $l_{2}$ is odd or even.

We first take $l_{2}$ odd. The fraction $(2 \beta-\nu) / 2 \nu$ will reduce to the form $\pm 1 / l_{2}$ only when $\nu$ is even. We may therefore assume

$$
\nu=2 k_{2} l_{2} \quad \text { and } \quad \beta=k_{2}\left(l_{2} \pm 2\right) \text {. }
$$

From (2), part I, $\S 1, q \beta$ takes the form $m \nu+\beta^{\prime}$, whence, from the preceding relations,

$$
\beta^{\prime}=q k_{2}\left(l_{2} \pm 2\right)-2 m k_{2} l_{2} .
$$

Comparing this equation with $\beta^{\prime}=k_{2}\left(l_{2} \pm 2\right)$, we have

whence

$$
q k_{2}\left(l_{3} \pm 2\right)-2 m k_{2} l_{2}=k_{2}\left(l_{2} \pm 2\right),
$$

$$
\pm q \pm 1=l_{2} m+(1-q) l_{2} / 2 \text {. }
$$

Hence $q \equiv \pm 1\left(\bmod . l_{2}\right)$.

It is evident that $l_{2}$ may equal 3 , since all values of $q$ not divisible by 3 satisfy the congruence $q \equiv \pm 1(\bmod .3)$.

On account of theorem III, the number of parameters $\omega$ cannot exceed 4 , that is, the number of values of $q$, greater than unity and less than $\nu$ which are relatively prime to $\nu$, must not exceed 3. Hence $\nu$ cannot exceed 12, and 
since $l_{2}$ is an odd factor of $\nu$, it cannot exceed 3 , except that $l_{2}$ may be infinite in case $\beta=\nu / 2$.

Next, take $l_{2}$ even and equal to $2 \lambda_{2}$. From (2) p. 444 , we may assume

$$
\nu=k_{2} \lambda_{2} \quad \text { and } \quad \beta=\frac{k_{2}\left(\lambda_{2} \pm 1\right)}{2}
$$

Accordingly

$$
q \beta=q k_{2} \frac{\left(\lambda_{2} \pm 1\right)}{2}=m \nu+\beta^{\prime}
$$

whence

$$
\beta^{\prime}=q k_{2} \frac{\left(\lambda_{2} \pm 1\right)}{2}-m k_{2} \lambda_{2} .
$$

Since $\beta$ and $\beta^{\prime}$ are required to satisfy the same condition (2), we have

$$
\frac{k_{2}\left(\lambda_{2} \pm 1\right)}{2}=q k_{2} \frac{\left(\lambda_{2} \pm 1\right)}{2}-m k_{2} \lambda_{2},
$$

whence $\pm q \pm 1=\lambda_{2}(1+2 m-q)$, or $q \equiv \pm 1\left(\bmod . \lambda_{2}\right)$. This congruence is satisfied by all allowable values of $q$ when $\lambda_{2}$ equals 2 or 3 , that is, when $l_{2}=4$ or 6 . The only other possible values for $\lambda_{2}$ are 4 and 6 , and for $\nu, 4,6,8$ and 12. The cases $\nu=4$ and 6 give nothing new. For $\nu=8, \lambda_{2}=4$, the condition $2 \beta=k_{2}\left(\lambda_{2} \pm 1\right)$ gives $\beta=3$ or 5 . Since $\beta^{\prime}$ satisfies the same relation, it can take no additional values. But for $\beta=3, q=3$, we find $\beta^{\prime}=1$. If $\nu=12, \lambda_{2}=4$, then $2 \beta=3(4 \pm 1)$ does not give an integer value for $\beta$. Thus it is seen that $\lambda_{2}=4$ is impossible. In a similar manner, it may be shown that $\lambda_{2}=6$ is also impossible. Hence the only possible values for $l_{2}$ are $3,4,6$.

Since the substitution $V_{1}$ has the fixed points $\left(\sigma^{-a-\beta}-1\right)$ and 0 , it may be written in the following form:

from which we deduce

$$
\frac{\omega_{1}^{\prime}-\sigma^{-a-\beta}+1}{\omega_{1}^{\prime}}=K \frac{\omega_{1}-\sigma^{-a-\beta}+1}{\omega_{1}} .
$$

$$
K=\sigma^{(a+\beta)}={ }^{2 \pi i(a+\beta) i \nu} \text {. }
$$

But, as stated for the substitution $S_{1}$, this must be of the form $e^{2 \pi i\left(p+1 / t_{3}\right)}$, whence

$$
\frac{\alpha+\beta i}{\nu}= \pm \frac{1}{l_{3}}+p
$$

Now from the inequalities $0<\alpha<\nu$, and $0<\beta<\nu$, we get $0<\left(\alpha_{T} \beta\right) / \nu<2$. Hence four values are possible for $\alpha+\beta$, namely,

$$
\frac{\alpha+\beta}{\nu}= \pm \frac{1}{l_{3}}+1, \quad \frac{\alpha+\beta}{\nu}=-\frac{1}{l_{3}}+2, \quad \frac{\alpha+\beta}{\nu}=\frac{1}{l_{3}} .
$$


The fraction $1 / l_{3}$ may be put in the more general form $k_{3} / l_{3} k_{3}$, whence we find $\nu=k_{3} l_{3}$. Then the four possible values for $\alpha+\beta$ are given in the equations

$$
\begin{cases}(a), & \alpha+\beta=k_{3}\left(l_{3} \pm 1\right), \\ (b), & \alpha+\beta=k_{3}\left(2 l_{3}-1\right), \\ (c), & \alpha+\beta=k_{3} .\end{cases}
$$

Since $q \alpha=m \nu+\alpha^{\prime}$, and $q \beta=n \nu+\beta^{\prime}$, where $m$ and $n$ are positive integers or zero, we have

$$
\begin{aligned}
& \left(a^{\prime}\right) \quad \alpha^{\prime}+\beta^{\prime}=q k_{3}\left(l_{3} \pm 1\right)-(m+n) \nu, \\
& \left(b^{\prime}\right) \quad \alpha^{\prime}+\beta^{\prime}=q k_{3}\left(2 l_{3}-1\right)-(m+n) \nu, \\
& \left(c^{\prime}\right) \quad \alpha^{\prime}+\beta^{\prime}=q k_{3}-(m+n) \nu .
\end{aligned}
$$

And as $\alpha+\beta$ and $\alpha^{\prime}+\beta^{\prime}$ must have the same form, four possibilities are to be considered.

First, suppose $\alpha+\beta=k_{3}$, then $\alpha^{\prime}+\beta^{\prime}=q k_{3}-(m+n) \nu$. But $\alpha^{\prime}+\beta^{\prime}$ must be one of the forms $(a),(b),(c)$ of (6). Taking the condition $(a)$, we get $q k_{3}-(m+n) \nu=k_{3}\left(l_{3} \pm 1\right)$, which by the use of $\nu=k_{3} l_{3}$, becomes $k_{3}(q \pm 1)=k_{3} l_{3}(m+n+1)$, whence $q \equiv \pm 1\left(\bmod . l_{3}\right)$. Taking the condition $(b)$, we have $q k_{3}-(m+n) \nu=k_{3}\left(2 l_{3}-1\right)$, which gives $q \equiv-1\left(\bmod . l_{3}\right)$. Taking condition (c), there results $q k_{3}-(m+n) \nu=k_{3}$, from which $q \equiv 1$ (mod. $\left.l_{3}\right)$.

Proceeding in like manner with the values of $\alpha+\beta$ given in (b) and (c) of $(6)$ we obtain the same conditions for $q$ as before. Therefore, all the numbers $q$ must satisfy the congruence $q \equiv \pm 1\left(\bmod . l_{3}\right)$.

It is evident that $l_{3}$ may equal $2,3,4,6$, since all the numbers $q$ not containing $2,3,4$, or 6 as a factor will satisfy the above congruence. If $l_{3}=5$, we obtain $\nu=5,10,15$, etc. But for these values of $\nu$ some of the numbers $q$ do not satisfy $q \equiv \pm 1(\bmod .5)$. It is not necessary to examine for values of $\nu$ greater than 10 , since the number of values of $q$ will then exceed 4 (see theorem III). Hence $l_{3}$ cannot equal 5. In the same way it can be shown that $l_{3}$ cannot equal any number greater than 6 .

The values $l_{1}=2, l_{2}=3,4,6$, and $l_{3}=2,3,4,6$, in those combinations only which satisfy the inequality

$$
\frac{1}{l_{1}}+\frac{1}{l_{2}}+\frac{1}{l_{3}}<1
$$

give us three sets, namely, $l_{1}=2, l_{2}=4, l_{3}=6 ; l_{1}=2, l_{2}=6, l_{3}=4$; $l_{1}=2, l_{2}=6, l_{3}=6$. Using the first set in connection with equations (4) and (6) of this section, and $\nu=k_{3} l_{3}$, we obtain the Riemann surface $W^{12}=(z-a)(z-b)^{9}(z-c)^{9}(z-d)^{5}$. For $\nu=12, q$ has the values 1, 5, 7, 
11, which are found to satisfy the congruence conditions, of $\S 3$, class 4 , part I. Thus the necessary conditions that the four $\omega$ 's may be linearly dependent are fulfilled. These, however, are not sufficient, as it is seen by actually working out the table of moduli that two of the $\omega$ 's are linearly independent. Hence this surface is to be excluded. In a similar manner using the second set of values for the $l$ 's, we obtain the Riemann surface $W^{12}=(z-a)(z-b)^{8}(z-c)^{8}(z-d)^{7}$, which must also be excluded, for the same reason as before. The third set of values for the $l$ 's gives the surface $W^{6}=(z-a)^{3}(z-b)^{2}(z-c)^{2}(z-d)^{5}$, which has already been treated (see footnote p. 425).

If $l_{2}$ equals infinity, we get the three sets $l_{1}=2, l_{2}=\infty, l_{3}=3$; $l_{1}=2, l_{2}=\infty, l_{3}=4 ; l_{1}=2, l_{2}=\infty, l_{3}=6$, which however give nothing new. Hence we conclude that no new cases of the kind sought for are found in Class 4.

\section{§2. Values of $l_{1}, l_{2}, l_{3}$ for class 5 .}

By compounding the transformations of this class there is obtained the transformation $T_{1}=V_{1}^{-1} S_{1}^{-1}$, namely,

$$
\omega_{1}^{\prime}=\frac{\omega_{1}+\sigma^{-a+\gamma}-\sigma^{\gamma}}{\left(\sigma^{-\gamma}-1\right) \omega_{1}+\sigma^{-a}+\sigma^{\gamma}-1} .
$$

By the method employed for substitution $V_{1}$ in Class 4, we obtain the following values for $K$, namely, for $T_{1}$,

$$
K=\sigma^{a+\gamma}=e^{2 \pi i(a+\gamma) / \nu},
$$

for $S_{1}$,

for $V_{1}$,

$$
\boldsymbol{K}^{\zeta}=\sigma^{\beta+\gamma}=e^{2 \pi i(\beta+\gamma) / \nu},
$$

$$
K=\sigma^{a+\beta}=e^{2 \pi i(a+\beta) / \nu} .
$$

There are sets of equations for $\alpha+\gamma, \beta+\gamma$, and $\alpha+\beta$, similar to those of $(6)$ of the previous section, where $l_{1}, l_{2}, l_{3}$ denote the $l$ for substitutions $T_{1}, S_{1}$, and $V_{1}$ respectively. The value of $\nu$ is given by the following equations,

$$
\begin{cases}(a) & \nu=l_{1} k_{1}, \\ (b) & \nu=l_{2} k_{2}, \\ (c) & \nu=l_{3} k_{3} .\end{cases}
$$

By the method of the preceding section it may be shown that $q$ must satisfy the congruence $q \equiv \pm 1\left(\bmod . l_{i}\right),(i=1,2,3)$, and that $l_{1}, l_{2}, l_{3}$ may each have only the values $2,3,4,6$. It will not be necessary to examine in this connection for values of $\nu$ greater than those in which there may be eight values of $q$ (see theorem IV). 
Taking only those combinations of the $l$ 's which satisfy the inequality (7) above, we obtain the equation of but one Riemann surface, namely,

$$
W^{24}=(z-a)(z-b)^{11}(z-c)^{19}(z-d)^{17},
$$

in which $l_{1}=2, l_{2}=4, l_{3}=6$. It may be shown that all the necessary conditions that the eight parameters may be linearly connected are satisfied. Whether they are actually so connected, we do not need to determine, since the $\omega$-group in this case would be $(0,3 ; 2,4,6)$ and functions belonging to this group can be expressed more simply by means of hyperelliptic theta functions.* If one of the $l$ 's is assumed equal to infinity, nothing new is obtained.

Therefore no new cases are found in Class 5.

Finally, in classes 2 and 3 the value of $\nu$ cannot be greater than 6 , as otherwise there would be more than one linearly independent $\omega$ according to theorem II. All the surfaces for $\nu$ equal to 6 or less have been examined and nothing new is found.

Rutakrs College, New BRUNSwick, N. J.

* See Hutchinson, On the functions of the group $(0,3 ; 2,6,6)$. 